\title{
A case control series for the effect of photobiomodulation in patients with low back pain and concurrent depression
}

\author{
Charles Philip Gabel ${ }^{1}$, Samuel R Petrie ${ }^{2}$, David Mischoulon ${ }^{2}$, \\ Michael R Hamblin ${ }^{3,4}$, Albert Yeung ${ }^{2}$, Lisa Sangermano ${ }^{2}$, Paolo Cassano ${ }^{2,5}$ \\ 1: Coolum Physiotherapy, Sunshine Coast, Queensland \\ 2: Harvard Medical School, Depression Clinical and Research Program, \\ Harvard University, Massachusetts General Hospital, Boston \\ 3: Wellman Center for Photomedicine, Massachusetts General Hospital, Boston, \\ Department of Dermatology, Harvard Medical School, Boston \\ 4: Harvard-MIT Division of Health Science and Technology, Cambridge MA 02139. \\ 5: Harvard Medical School, Center for Anxiety and Traumatic Stress Disorders, \\ Harvard University, Massachusetts General Hospital, Boston
}

\begin{abstract}
Background and aims: To present incidental findings in patients with low back pain (LBP) who received photobiomodulation (PBM) administered to the back and thighs as an adjunct to physical therapy (PT) and then experienced improvement in concurrent depression.

Materials and methods: Five outpatients with LBP and concurrent self-reported depression were treated for LBP over five weeks with PT (5-sessions) and concurrent PBM (final 3-sessions), and retrospectively matched to five control patients treated with PT alone (5-sessions). The PBM device emitted light at $850 \mathrm{~nm}$ and $660 \mathrm{~nm}$ with an irradiance of $100 \mathrm{~mW} / \mathrm{cm}^{2}$ and fluence of $3 \mathrm{~J} / \mathrm{cm}^{2}$ on 12 symmetrical posterior sites (thoracic, lumbar and thighs) for $30 \mathrm{sec} / \mathrm{site}$.

Results: Both groups had non-significant differences in all baseline scores, except for higher functional status (ARGS) in the PBM-group $(33.6 \pm 12.2$ vs.18.6 $\pm 3.6, t(8)=2.638, p=0.030)$. After treatment, the mean decrease in depression scores (OMSQ-12 item \#6) was significantly larger in the PBM-group (43.0 \pm 22.0 vs. $8.0 \pm 5.7, t(8)=3.449, p=0.009$ ). Improvement in functional status (ARGS) in the PBM-group was similar to that in the controls $(42.0 \pm 13.5$ vs. $43.4 \pm 11.1, t(8)=0.179, p=0.862)$, suggesting group differences in antidepressant effect were independent of functional status improvement.

Conclusions: This preliminary investigation suggests that an antidepressant effect may result from PBM to the back and thighs in patients with LBP and concurrent depression.
\end{abstract}

Key words: Depression - photobiomodulation $\cdot$ near infrared and red light $\cdot$ low back pain • low level laser therapy

\section{Introduction}

Patients with spine-related problems, ${ }^{1)}$ chronic-pain or chronic orthopedic disease ${ }^{2)}$ and particularly low back pain (LBP), ${ }^{3)}$ frequently suffer with concurrent major depressive disorder (MDD). When pain and depression are comorbid, causality is difficult to ascertain as the chronic LBP may lead to depression, and physical pain is a com-

\footnotetext{
Addressee for Correspondence:

Paolo Cassano, MD, PhD

1 Bowdoin Square, $6^{\text {th }}$ Floor

Massachusetts General Hospital

Boston, MA 02114

$1-617-643-9622$

mon symptom of depression. ${ }^{4)}$ Regardless of causality, comorbid depression is often associated with more intense pain ${ }^{5)}$ and worsens the prognosis of LBP. ${ }^{6}$ Despite the prognostic relevance in LBP, depression is under-recognized by primary care practitioners. ${ }^{6,7)}$ Consequently, a brief screening for depression in patients complaining of LBP is recommended, but there is controversy as to the minimum number of questions required. ${ }^{8-10)}$ Furthermore, the use of specific strategies such as a multidisciplinary rehabilitation program-addressing a combination of physical, psychological, social or work-related factors-

Received date: January 31st, 2018 Accepted date: May 29th, 2018 
did not demonstrate an additional effect on depressive symptoms when compared to physical rehabilitation alone according to a Cochrane review. ${ }^{4}$

Depression has been linked to a decreased level of mitochondrial respiration in blood platelets. ${ }^{11)}$ Consequently, blood cells have been proposed as a target for systemic therapy for depression. ${ }^{12)}$ This theory suggests possible involvement of whole blood in the pathophysiology of depression. Photobiomodulation (PBM) - an FDA-approved treatment for somatic pain-is based on the boosting of ATP production by near infra-red (NIR) or red light (delivered by either a laser or light emitting diode (LED) source) ${ }^{13,14)}$ via stimulation of the mitochondrial respiratory chain. ${ }^{15,16)}$ In a few published studies, transcranial PBM and laser acupuncture have shown benefit in patients with depression; ${ }^{17-21)}$ however, there is currently no literature indicating whether PBM applied to large areas of peripheral skin (as opposed to the head or to small acupuncture and trigger points) improves depression.

This case series presents incidental findings observed in two separate groups of patients with LBP. In the first group, patients with MDD as diagnosed by their General Practitioner (GP) who scored highly for depression severity, received PBM therapy using NIR and red wavelength light from a LED source. In the second group, patients with similar high depression scores but no clinically-ascertained diagnosis of MDD were not treated with PBM. Both groups received standardized physical therapy including a home exercise therapy program. ${ }^{22)}$ The aim of the study was to retrospectively test whether NIR and red PBM, applied to large areas of skin on the back and thighs, decreased the severity of depression.

\section{Materials and methods}

\section{Patient Selection}

By means of retrospective, post-hoc chart reviews, two homogenous groups of five outpatients each were formed in two separate case series: a treatment group and a control group. The first group was formed by consecutives patients who received PBM [to the back and posterior thighs] as an adjunct therapy after two initial physical therapy (PT) sessions. The second group subjects were selected as matched controls to the first group based on age, gender, presenting symptoms and level of reported depression. All patients in both groups had been receiving PT after presenting with primary LBP of a musculoskeletal source and with self-reported depression. The entry depression severity was at least moderate -except for one pair of subjects who had mild to moderate depression- based on the Orebro Musculoskeletal Screening Questionnaire, OMSQ-12 item \#6 with a cut off $>60 \%$ (where $100 \%$ is the highest degree of depression). This question format and scaling, expressed as a percentage, was comparable to the one-question depression scale used and determined by Reme ${ }^{9,10)}$ and to that by Haggman and colleagues. ${ }^{6}$ This study proposal was submitted to an Institutional Review Board (IRB), the Partners Human Research Committee (PHRC). The PHRC determined that the study did not meet criteria for human

Table 1: Demographics, Functional Status (determined by ARGS score), and Depression (determined by score on item 6 of the OMSQ-12) at different time points throughout the study for both active (NIR) and control (CTRL) group participants.

\begin{tabular}{|c|c|c|c|c|c|c|c|c|c|}
\hline \multirow[b]{2}{*}{ Condition } & \multicolumn{3}{|c|}{ Demographics } & \multicolumn{2}{|c|}{$\begin{array}{l}\text { Week } 1 \text { (Prior to } \\
1^{\text {st }} \text { PT treatment) }\end{array}$} & \multicolumn{2}{|c|}{$\begin{array}{c}\text { Week } 2 \text { (Prior to } \\
1^{\text {st }} \text { PBM add-on treatment) }\end{array}$} & \multicolumn{2}{|c|}{$\begin{array}{c}\text { Week } 5 \text { (After end of } \\
\text { treatments) }\end{array}$} \\
\hline & PT ID & Age & Sex & ARGS & OMSQ-12 Item 6 & ARGS & OMSQ-12 Item 6 & ARGS & OMSQ-12 Item 6 \\
\hline \multirow{5}{*}{ NIR } & 1 & 65 & M & 40 & 65 & 51 & 60 & 73 & 35 \\
\hline & 2 & 75 & M & 37 & 75 & 60 & 65 & 87 & 30 \\
\hline & 3 & 57 & $\mathrm{~F}$ & 41 & 45 & 54 & 40 & 73 & 20 \\
\hline & 4 & 56 & $\mathrm{~F}$ & 12 & 65 & 28 & 55 & 74 & 30 \\
\hline & 5 & 48 & $\mathrm{~F}$ & 38 & 100 & 49 & 80 & 71 & 20 \\
\hline \multirow{5}{*}{ CTRL } & 6 & 62 & M & 19 & 80 & - & - & 71 & 70 \\
\hline & 7 & 78 & M & 21 & 60 & - & - & 46 & 60 \\
\hline & 8 & 55 & $\mathrm{~F}$ & 23 & 50 & - & - & 75 & 45 \\
\hline & 9 & 52 & $\mathrm{~F}$ & 14 & 60 & - & - & 60 & 50 \\
\hline & 10 & 45 & $\mathrm{~F}$ & 16 & 100 & - & - & 58 & 85 \\
\hline
\end{tabular}

(-) CTRL group participants did not receive an assessment at week 2. 
subjects research because no identifiable private information was available to the individuals conducting the research and all data was a retrospective analysis of individual cases grouped to form a series. Thus, it was not necessary to subsequently seek and obtain informed consent from the patients who were selected in and analyzed for this study.

The PBM group (group 1) comprised patients who had been diagnosed as clinically depressed and were also taking Selective Serotonin Re-uptake Inhibitor (SSRI) antidepressants as prescribed by their medical practitioner. Each participant stated they had been on an antidepressant regimen for at least 12 months preceding the onset of the episode of LBP. Each patient was treated for pain with PT alone, including manual and exercise therapy, for two sessions over two weeks and subsequently with PT plus PBM for three sessions over three weeks. The therapeutic choice to concurrently add PBM at the third session was based on the patients' history of concurrent clinical depression, the fact that no change in the depressive symptoms had resulted from the two previous PT only sessions, and in consideration of the reported benefits of PBM on mood, anxiety and pain. The control group (group 2) was selected retrospectively through a post-hoc matching of the participants in group 1 for age, gender, diagnosis of LBP, treatment with PT, and the presence of depression of similar severity, as measured on the OMSQ-12 item \#6. Besides the lack of PBM treatment, group 2 differed from group 1 because, although the subjects had the same measured baseline level of depression-as measured on the OMSQ-12 item \#6-they had not been diagnosed with MDD by a GP (self-referrals to PT clinic) nor had they been prescribed nor were taking any antidepressant medications. Consequently, the physical therapist only augmented PT with PBM treatment for patients in LBP (group 1) whose comorbid depression was signaled by their antidepressant medication.

\section{Assessment of Depression and of Functional Outcome}

Depression was assessed using a one-question depression scale ${ }^{6,9)}$ extracted from the Short Form 12-item version of the Orebro Musculoskeletal Screening Questionnaire (OMSQ-12) (minimal detectable change- $\mathrm{MDC}=$ $20 \%{ }^{23)}$-an established questionnaire for the assessment of biopsychosocial-risk profile. The question extracted was Question \#6, scored on an 11-point Likert Scale anchored from $0=$ "Not at all' to $10=$ "Extremely" and stated: "During the past 2-3 days, rate how 'depressed' or 'down' you have felt". The conversion to a percentage scale was made by multiplying by a factor of 10 .

The anatomical region-specific functional outcome was measured using a 'Cloud' based decision support software system-Advise Rehab Software (MDC $\left.=5.0 \%) .{ }^{24}\right)$ This system used a Tablet-PC or I-Pad, as an interface with the patient, to collect responses in the following categories: 1) overall perceived functional status; 2) participation in everyday activities or duties; 3) pain; 4) spine regional functional status (on a 10-item self-reported outcome-PRO); 5) collateral indicators of daily function (5item patient specific index of self-selected items). ${ }^{25-27)}$ The 5 -domain scores were integrated by an algorithm that produced a single score from 0 to 100 on functional status, the Advise Rehab Global Scale (ARGS: 0 = Worst possible function, $100=$ Maximum or pre-injury function). ${ }^{24,28-30)}$

For group 1, all measurements were made on three occasions: at baseline, at week two, following the first two PT sessions, and at discharge (week five) — following the three additional manual therapy sessions with the adjunct PBM. For group 2, the same measurements were conducted at baseline and at discharge (week five), after completion of five manual therapy sessions.

\section{Intervention}

Manual therapy consisted of spinal mobilization, massage, and the use of home exercises for range of movement, strength and core control. ${ }^{6,31-33)}$ Both groups received a total of five manual therapy sessions. The therapist was a post-graduate Certified Sports Physical Therapist and Manual Diagnostic Therapist-McKenzie (CPG).

The PBM was delivered by the same therapist (CPG) with a Thor-UK:DDII, LED-104 instrument (see Figure 1). This device delivers both red and NIR continuous wave light and comprises an integrated cluster-probe containing LEDs with the following wavelength and individual LED power values, respectively: $660 \mathrm{~nm}$ ( 56 probes each emitting $10 \mathrm{~mW}$ ) and $850 \mathrm{~nm}$ (48 probes each emitting $30 \mathrm{~mW}$ ). The total power was 2 Watts, roughly $75 \%$ NIR and $25 \%$ red light. The spot-size of the probe totaled $20 \mathrm{~cm}^{2}$ (single spot-size $1 / \mathrm{e}^{2}=0.2 \mathrm{~cm}^{2}$ ). The treatment window (total area treated) was $240 \mathrm{~cm}^{2}$ and included 12 symmetrical bilateral sites $\left(20 \mathrm{~cm}^{2}\right.$ per site):

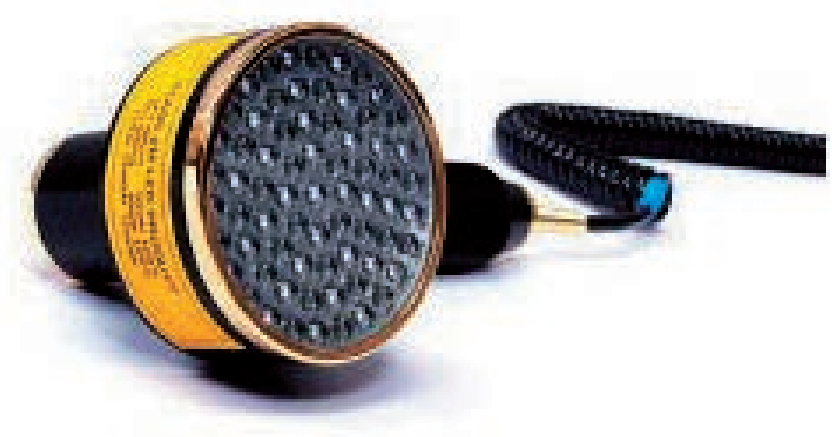

Figure 1: Image of Thor-UK:DDII, LED-104 instrument 
eight thoracic and four posterior-thigh sites (see Figure 2). The average irradiance was $100 \mathrm{~mW} / \mathrm{cm}^{2}$ and fluence was $3 \mathrm{~J} / \mathrm{cm}^{2}$. The entire PBM session lasted $6 \mathrm{~min}(30 \mathrm{sec} / \mathrm{site}$ for a total of 12 sites); the NIR and red light were delivered over a 14-18 day period for a total of three treatments at direct skin contact. The dose per session was $720 \mathrm{~J}(60 \mathrm{~J} / \mathrm{site})$.

\section{Analyses}

Group 1 and 2 were compared post-hoc to determine the effect of PBM on the reported levels of depression as well as on overall functional status. Baseline demographic variables and severity scores were compared between the two groups by independent samples t-test for equality of means and by Fisher's Exact test, for continuous and dichotomous variables, respectively (2-tailed significance at $\mathrm{p} \leq 0.05$ ). Mean changes in depression scores (OMSQ12 item \#6) and in function (ARGS total score) from baseline to discharge were compared between the two groups by independent samples t-test for equality of means (2-tailed significance at $\mathrm{p} \leq 0.05$ ). A secondary analysis was conducted within the PBM group (1), to compare the mean changes in depression scores, within the same subjects, pre- and post- PBM, by paired samples t-test. For graphic representation only, within each group, the mean total scores for depression and function were compared from baseline to discharge by paired sample t-test. Twotailed significance for all five secondary analyses was set at $\mathrm{p} \leq 0.01$ after adopting a Bonferroni correction (SPSS Inc. Released 2008. SPSS Statistics for Windows, Version 17.0. Chicago: SPSS Inc.).

\section{Results}

Gender and age were similar for the PBM treatment group and for the matched control group (60\% women in both groups; age $60.2 \pm 10.2$ vs. $58.4 \pm 12.5$ years) (statistics not

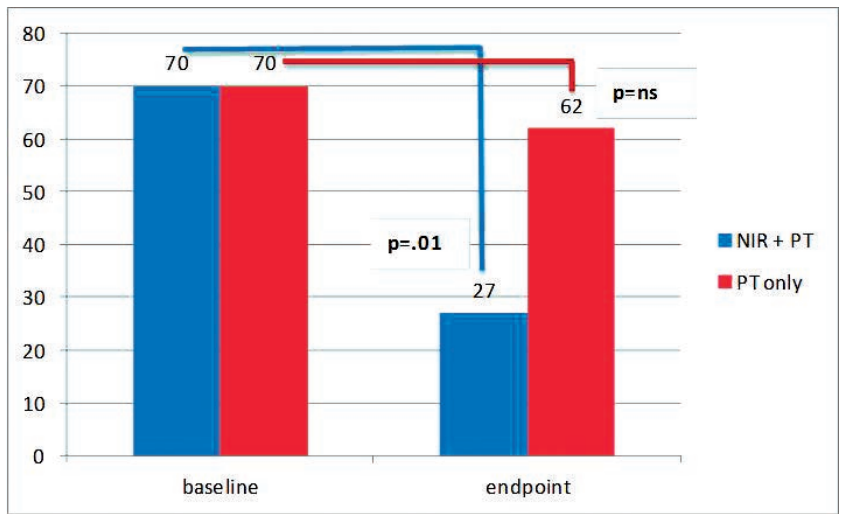

Figure 3: Baseline and final scores of depression in patients treated with and without photobiomodulation (near-infrared radiation (NIR) and red light) for back pain shown; $p$-value not significant) (see Table 1). The two groups showed identical baseline depression severity (OMSQ-12 item \#6 at baseline: $70.0 \pm 20.0$; Figure 3); however, functional status was significantly worse in the control group (PBM vs. Controls; ARGS total score at baseline $33.6 \pm 12.2$ vs. $18.6 \pm 3.6, t(8)=2.638, p=0.030$;

Figure 4). All but one subject per group had at least moderate depression severity; the remainder mild to moderate severity.

The change in depression scores from baseline to discharge (after the $5^{\text {th }}$ session) was significantly greater in the PBM group than in the control group (decrease in OMSQ-12 item \#6 baseline - endpoint: $43.0 \pm 22.0$ vs. $8.0 \pm 5.7, t(8)$ $=3.449, p=0.009$; Figure 3). Notably, both groups im-

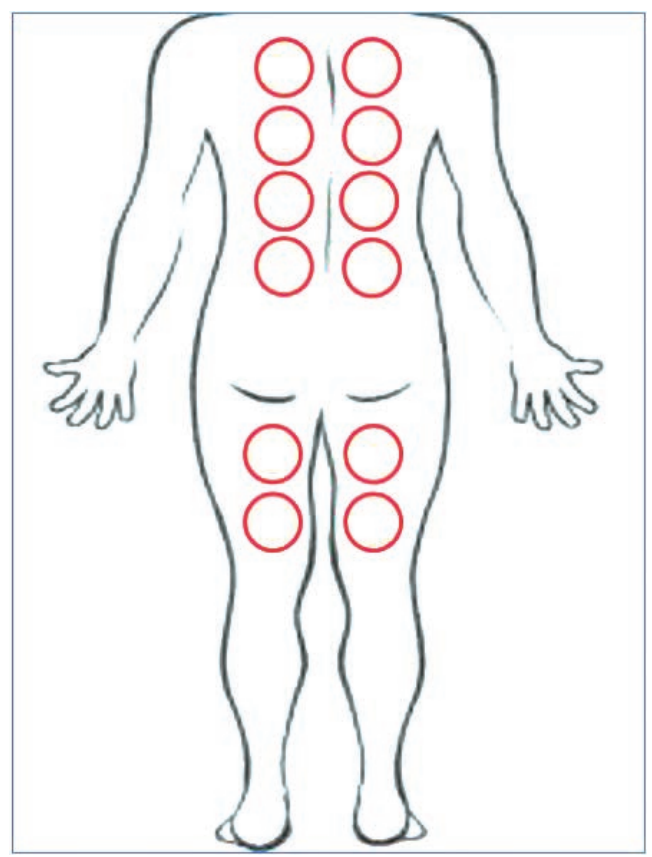

Figure 2: Areas Treated with Thor 104 cluster

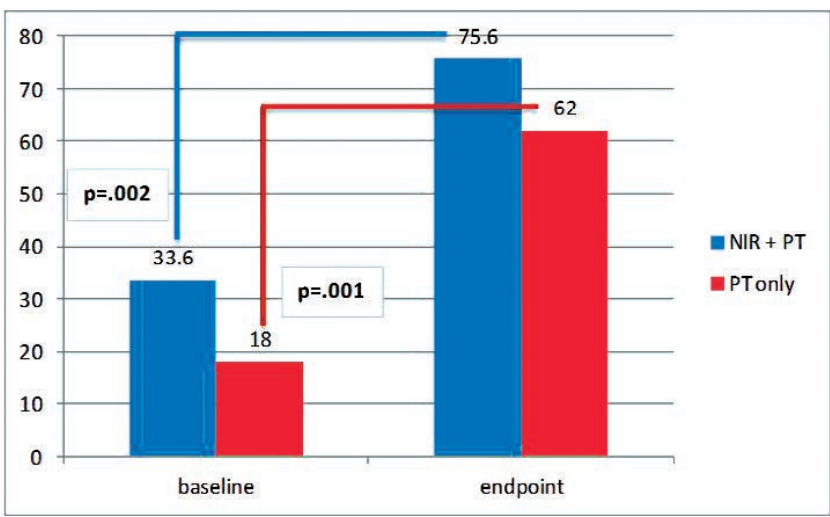

Figure 4: Baseline and final scores of functional status in patients treated with and without photobiomodulation (near-infrared radiation (NIR) and red light) for back pain 
proved significantly regarding functional status (Figure 4), and the change in functional status in the PBM group was similar to the change observed in the control group (increase in ARGS total score baseline - endpoint: $42.0 \pm 13.5$ vs. 43.4 $\pm 11.1, t(8)=0.179, p=0.862$; Figure 4). This suggests that the group differences for the antidepressant effect were independent of improvements in function and pain. See also Figure 3 and $\mathbf{4}$, depicting the histograms of the mean total scores for depression and function, respectively, at baseline and endpoint.

When a secondary analysis was performed within the PBM treatment group, the change in depression during the actual PBM phase (sessions \# 3-5) was significantly greater than the change in depression during prePBM phase (sessions \# 1-2; change in OMSQ-12 item \#6 pre-session \#3 -endpoint $33.0 \pm 16.0$ vs. item \#6 baseline - post-session $\# 210.0$ $\pm 6.1, t(4)=4.960, p=0.008)$.

\section{Discussion}

To our knowledge this is the first study to suggest -based on incidental findings- that PBM delivered to large areas of the skin may exert an antidepressant effect in subjects reporting LBP.

Two small, open studies have shown the antidepressant effect of transcranial PBM (NIR $810 \mathrm{~nm}$ and 808 $\mathrm{nm}$ ) in patients suffering from major depressive disorder: both studies suggested a robust, though non-sustained, response rate to NIR. ${ }^{20,17)}$ Additional open studies have also reported an antidepressant and anxiolytic effect in subjects treated with transcranial PBM for traumatic brain injury (TBI). ${ }^{34-36)}$ The primary mechanism hypothesized to explain the therapeutic effects of transcranial PBM is the boosting of brain metabolism, mediated by direct potentiation of the mitochondrial respiratory chain. ${ }^{37,38)}$

Interestingly, an Australian group has also used laser NIR (808 nm) light, though non-transcranially, delivered to acupuncture-points to treat major depressive disorder. The treatment focused on five primary depression acupoints and the total amount of energy delivered during each session ranged from 3 to $5 \mathrm{~J} .{ }^{18,19)}$ Although this dose of light was apparently low, the total energy delivered over the skin per session was sufficient to produce significant antidepressant effects in two double-blind trials using up to 12 sessions over eight weeks. A placebo-controlled randomized study from a Turkish group examined the effect of laser (904 nm) light-delivered to at least eleven tender points-in patients suffering from fibromyalgia but not MDD. In this trial, the light delivered to multiple circumscribed skin areas (each of $1 \mathrm{~cm}^{2}$ ) had a significant effect on subthreshold depressive symptoms despite the use of low fluence $\left(2 \mathrm{~J} / \mathrm{cm}^{2}\right.$ for a total of 10 sessions over two weeks). ${ }^{39)}$ These clinical effects might result from PBM of the peripheral and central nervous system, considering that acupoints-and possibly tender points- have been shown to overlie major neuronal bundles. ${ }^{40)}$

Our findings suggest that PBM to the skin (NIR $850 \mathrm{~nm}$ and red 660nm) might indeed exert an antidepressant effect, even when the nervous system is not directly or specifically targeted, as opposed to PBM administered to the skull or to acupoints or tender points. The assumption is that NIR and red light, delivered to large areas of the skin and to the embedded vasculature, might significantly affect systemic processes such as hypometabolism, inflammation, and oxidative stress, implicated in the pathophysiology of depression. ${ }^{12,13)}$

\section{Limitations and Strengths}

Our study has several limitations. 1). The use of antidepressant medications in the PBM group is a confounding factor. It is possible, but unlikely, that the antidepressant effect in this latter group was due to the medications and not to the PBM. While we do not have sufficient information on concomitant treatments to rule this out, patients had been on antidepressants for at least 12 months and were still clinically depressed. Moreover, within the PBM group there was a statistically-significant antidepressant benefit reported following the PBM sessions, when compared to the prior phase of only manual PT. 2) The extracted depression measurement questionnaire used in this study has not been separately validated, whereas the original scale has been. However, our screening question for depression is similar in wording and scoring to the question used and validated by Reme and colleagues 9) for patients with LBP and depression, and also to that used by Haggman and colleagues. ${ }^{6}$ The single-item screening question used by Reme ${ }^{9)}$ was extracted from the Subjective Health Complaint Inventory (SHCI ${ }^{10)}$ and compared with the Hospital Anxiety and Depression Scale ${ }^{41)}$ and the Hopkins Symptom Checklist-25 ${ }^{42)}$ and is sensitive to depression severity. 3) Our open, retrospective case-series study is subject to limitations inherent to its design including patients' expectation that an additional device-based intervention might help them (placebo effect) and including the lack of randomization with a rigorously designed and deployed sham comparison. However, it is unlikely that the psychological benefits reported by the PBM-group were secondary to improvements in physical function and pain, since these latter measurements improved to a similar extent in both groups, with and without PBM. 4) The lack of a documented clinical diagnosis of MDD in the control group might invalidate our comparison. Still, the high comorbidity between LBP and clinical depression and the endorsement of equally severe depressed mood in both study groups suggest that our controls might be adequate.

The primary strength of this case-series is that it reports incidental, clinically-significant findings which were common to all patients receiving PBM. The latter is the single common intervention that might have contributed 
to the changes in depression: the use of NIR and red light over a large area of body skin providing potential systemic effects. The results suggest that the PBM sessions might have improved symptoms of depression in five patients; as a statistically significant difference was found both, within the PBM-treated group following the addition of the PBM to the PT regime, and between patients who did and did not received the PBM sessions.

\section{Conclusions}

This clinical case-series presents incidental findings on the effects of PBM-therapy on problematic LBP patients with concurrent depression. The use of PBM to large areas of the skin might have beneficial effects as part of a multi-modal treatment approach specifically targeted to depressed patients. However, in this case-series report, PBM cannot be identified as the single-agent producing the psychological benefit, due to the above limitations in the design, and the findings must therefore be considered preliminary. Prospective randomized controlled trials are necessary to better characterize the efficacy of PBM in the population of our study.

\section{References}

1: Altuğ F, Kavlak E, Kurtca MP, Ünal A, Cavlak U:Comparison of pain intensity, emotional status and disability level in patients with chronic neck and low back pain. J Back Musculoskelet Rehabil, 2015; 28(3):505-508.

2: McKillop AB, Carroll LJ, Battié MC: Depression as a prognostic factor of lumbar spinal stenosis: a systematic review. Spine J, 2014; 14:837-846.

3: Mandic N: Low back pain and depression. Eur Psychiatry, 1997; 12:186.

4: Kamper SJ, Apeldoorn AT, Chiarotto A, Smeets RJ, Ostelo RW, et al: Multidisciplinary biopsychosocial rehabilitation for chronic low back pain. Cochrane Database Syst Rev, 2014; 2:CD000963.

5: Main CJ, Wood PL, Hollis S, Spanswick CC, Waddell G: The Distress and Risk Assessment Method: a simple patient classification to identify and evaluate the risk of poor outcome. Spine (Phila Pa 1976) 1992; 17:42-52.

6: Haggman S, Maher CG, Refshauge KM: Screening for symptoms of depression by physical therapists managing low back pain. Phys Ther, 2004; 84:1157-1166.

7: Thapar A, Hammerton G, Collishaw S, Potter R, Harold G, et al: Detecting recurrent major depressive disorder within primary care rapidly and reliably using short questionnaire measures. Br J Gen Pract, 2014; 64:e31-37.

8: Mitchell AJ, Coyne JC: Do ultra-short screening instruments accurately detect depression in primary care?: A pooled analysis and meta-analysis of 22 studies. Br J Gen Pract, 2007; 57:144-151.

9: Reme SE, Eriksen HR: Is one question enough to screen for depression? Scand J Public Health, 2010; 38:618-624.

10: Reme SE, Lie SA, Eriksen HR: Are 2 questions enough to screen for depression and anxiety in patients with chronic low back pain? Spine (Phila Pa 1976) 2014; 39:E455-462.

11: Hroudová J, Fišar Z, Kitzlerová E, Zvěřová M, Raboch J: Mitochondrial respiration in blood platelets of depressive patients. Mitochondrion, 2013; 13:795-800.

12: Sommer AP, Trelles MA: Light pumping energy into blood mitochondria: a new trend against depression? Photomed Laser Surg, 2014; 32:59-60.

13: Chung H, Dai T, Sharma SK, Huang YV, Carroll JD, et al: The nuts and bolts of low-level laser (light) therapy. Ann Biomed Eng, 2012; 40:516-533.

14: Smith KC: Light and life: The photobiological basis of the therapeutic use of radiation from lasers. International Laser Therapy Association Conference. Osaka, 1990:130-141.

15: Karu TI: Photobiology of low power laser effects. Health Physics 1989; 56:691-704.

16: Liebert AD, Bicknell BT, Adams RD: Protein conformational modulation by photons: a mechanism for laser treatment effects. Med Hypotheses, 2014; 2:275-281.

17: Schiffer F, Johnston AL, Ravichandran C, Polcari A, Teicher $\mathrm{MH}$, et al: Psychological benefits 2 and 4 weeks after a single treatment with near infrared light to the forehead: a pilot study of 10 patients with major depression and anxiety. Behav Brain Funct, 2009; 8:46.

18: Quah-Smith I, Smith C, Crawford JD, Russell J: Laser acupuncture for depression: a randomised double blind controlled trial using low intensity laser intervention. J Affect Disord, 2013; 148:179-187.

19: Quah-Smith JI, Tang WM, Russell J: Laser acupuncture for mild to moderate depression in a primary care setting--a randomised controlled trial. Acupunct Med, 2005; 23:103111.

20: Cassano P, Cusin C, Mischoulon D, Hamblin MR, De Taboada, et al: Near-Infrared Transcranial Radiation for Major Depressive Disorder: Proof of Concept Study. Psychiatry J, 2015; 2015:352979.

21: Cassano P, Petrie S, Hamblin M, Henderson TA, Iosifescu DVHenderson: Review of Transcranial Photobiomodulation for Major Depressive Disorder: Targeting Brain Metabolism, Inflammation, Oxidative Stress, and Neurogenesis: Neurophotonics, 2016; 3(3):031404

22: Wellington $\mathrm{J}$ : Noninvasive and alternative management of chronic low back pain (efficacy and outcomes). Neuromodulation, 2014; 17 Suppl 2:24-30.

23: Gabel CP, Melloh M, Yelland M: The shortened Örebro Musculoskeletal Screening Questionnaire: evaluation in a work-injured population. Man Ther, 2013; 18:378-385.

24: Advise Rehab. Advise Rehabilitation, decision support software. Coolum Beach, Queensland: Advise Rehabilitation Pty Ltd 2013

25: Stratford P, Gill C, Westaway M, Binkley J: Assessing disability and change on individual patients: a report of a patient specific measure. Physiother Ca, 1995:58-63.

26: Westaway MD, Stratford PW, Binkley JM: The patient-specific functional scale: validation of its use in persons with neck dysfunction. J Orthop Sports Phys Ther, 1998; 27:331-338.

27: Gabel CP, Michener LA, Burkett B, Neller A: The Upper Limb Functional Index: development and determination of reliability, validity, and responsiveness. J Hand Ther, 2006; 19:328-348; quiz 349 .

28: Gabel C, Bardin L, Burkett B, Neller A: Intergrating injury screening with measurement and monitoring: a conceptual approach using a patient global assessment of the body and 
limbs scale. South African Journal of Physiotherapy, 2006:28.

29: Gabel C. Musculoskeletal screening and regional self report outcome measures: development and validation of clinical tools and investigation of a quantifiable link (between screening and regional measures). ROAR - Roadmap of Australian Research, 2006: http://www.adviserehab.com

30: Gabel C, Melloh M, Burkett B, Neller A, Yelland M: Measuring delayed recovery in musculoskeletal injuries: Primary Health Care Research Information Service, 2014: http:// www.phcris.org.au/phcred/snapshot/2014/story11.php.

31: Cherkin DC, Deyo RA, Battié M, Street J, Barlow W: A comparison of physical therapy, chiropractic manipulation, and provision of an educational booklet for the treatment of patients with low back pain. N Engl J Med, 1998; 339:10211029.

32: Mikhail C, Korner-Bitensky N, Rossignol M, Dumas JP: Physical therapists' use of interventions with high evidence of effectiveness in the management of a hypothetical typical patient with acute low back pain. Phys Ther, 2005; 85:1151-1167.

33: Poitras S, Blais R, Swaine B, Rossignol M: Management of work-related low back pain: a population-based survey of physical therapists. Phys Ther, 2005; 85:1168-1181.

34: Naeser MA, Saltmarche A, Krengel MH, Hamblin MR, Knight JA: Improved cognitive function after transcranial, light-emitting diode treatments in chronic, traumatic brain injury: two case reports. Photomed Laser Surg, 2011; 29:351-358.

35: Naeser MA, Zafonte R, Krengel MH, Martin PI, Frazier J, et al: Significant improvements in cognitive performance post-transcranial, red/near-infrared light-emitting diode treatments in chronic, mild traumatic brain injury: open-protocol study. J Neurotrauma, 2014; 31:1008-1017.

36: Morries LD, Cassano P, Henderson TA: Treatments for traumatic brain injury with emphasis on transcranial near-infrared laser phototherapy. Neuropsychiatr Dis Treat, 2015; 11:2159-2175.

37: Mochizuki-Oda N, Kataoka Y, Cui Y, Yamada H, Haya M, et al: Effects of near-infra-red laser irradiation on adenosine triphosphate and adenosine diphosphate contents of rat brain tissue. Neurosci Lett, 2002; 323:207-210.

38: Oron U, Ilic S, De Taboada L, Streeter J: Ga-As (808 nm) laser irradiation enhances ATP production in human neuronal cells in culture. Photomed Laser Surg, 2007; 25:180-182.

39: Gür A, Karakoc M, Nas K, Cevik R, Sarac J, et al: Effects of low power laser and low dose amitriptyline therapy on clinical symptoms and quality of life in fibromyalgia: a single-blind, placebo-controlled trial. Rheumatol Int, 2002; 22:188-193.

40: Zhou W, Benharash P: Effects and mechanisms of acupuncture based on the principle of meridians. J Acupunct Meridian Stud 2014; 7:190-193.

41: Zigmond AS, Snaith RP: The hospital anxiety and depression scale. Acta Psychiatr Scand, 1983; 67:361-370.

42: Derogatis LR, Lipman RS, Rickels K, Uhlenhuth EH, Covi L: The Hopkins Symptom Checklist (HSCL): a self-report symptom inventory. Behav Sci, 1974; 19:1-15.

\section{Acknowledgments}

We would like to acknowledge the Brain \& Behavior Research Foundation for providing salary support for Dr. Cassano (NARSAD Young Investigator Award). The sponsor was not involved in data collection and analysis or manuscript preparation and submission. 